\title{
Diacronie
}

Studi di Storia Contemporanea

$N^{\circ} 24,4 \mid 2015$

Le dittature militari: fisionomia ed eredità politica

\section{John Newsinger, Il libro nero dell'Impero britannico}

Jacopo Bassi

\section{(2) OpenEdition}

\section{Journals}

\section{Edizione digitale}

URL: http://journals.openedition.org/diacronie/3600

DOI: 10.4000/diacronie.3600

ISSN: 2038-0925

\section{Editore}

Association culturelle Diacronie

\section{Notizia bibliografica digitale}

Jacopo Bassi, « John Newsinger, Il libro nero dell'Impero britannico », Diacronie [Online], № 24, 4 | 2015, documento 21, online dal 29 décembre 2015, consultato il 10 décembre 2020. URL : http:// journals.openedition.org/diacronie/3600; DOI : https://doi.org/10.4000/diacronie.3600 


\section{Diacronie}

N. 24 | 4|2015 Le dittature militari: fisionomia ed eredità politica

20/

\section{RECENSIONE:}

\section{John NEWSINGER, Il libro nero dell'Impero britannico, Palermo, 21 editore, 2015, 387 pp.}

a cura di Jacopo BASSI *

John Newsinger, professore alla Bath Spa University, è un noto intellettuale inglese di orientamento marxista. Spesso controverso, il suo lavoro ha rappresentato un importante contributo della critica di sinistra alla storia moderna britannica e americana, in particolare alla luce dei recenti eventi politici e militari (ad esempio la "Guerra al terrorismo").

Nel corso della sua carriera, l'autore ha posto diverse domande incisive sulle strutture consolidate del potere - lo Stato, le questioni militari e quelle commerciali indagando le correnti intellettuali e le trame intessute dai centri di potere, scatenando spesso, con le sue ricerche, controversie e polemiche.

L'approccio marxista alla storia politica britannica, tuttavia, ha fornito un quadro intellettuale che è stato utile per migliorare la comprensione delle dinamiche dell'impero britannico. Newsinger, infatti, si è concentrato sulla violenza prodotta nel cuore degli imperi e ha sfidato alcuni testi che hanno trattato trionfalmente la storia britannica del XX secolo.

L’interesse del professor Newsinger per la ricontestualizzazione della percezione pubblica del passato imperiale britannico risale all'epoca precedente al suo ingresso nella Bath Spa University, nel 1992. Le sue ricerche, hanno avuto inizio con gli studi sul movimento repubblicano irlandese nel corso del XIX e XX secolo, e sono successivamente approdate alla pubblicazione di tre importanti monografie, edite quando Newsinger occupava già il ruolo di Lecturer/Senior Lecturer - e dal 2005 di professore - alla Bath Spa University ${ }^{1}$. La prima di queste è Orwell Politics², edito nel

\footnotetext{
${ }^{1}$ Oltre alle tre monografie menzionate più sotto, Newsinger è autore di: NEWSINGER, John, The Fenianism in mid-victorian Britain, London-Boulder, Pluto Press, 1994; ID., Dangerous men : the SAS and popular culture, London-Chicago, Pluto Press, 1997; ID., Rebel City: Larkin, Connolly and the Dublin Labour Movement, London, Merlin, 2004; ID., Fighting back : the
} 
1999 e ristampato nel 2001, nel 2003 e nel 2006: si tratta di uno studio sui convincimenti politici socialisti di George Orwell; i convincimenti politici dello scrittore britannico sono contestualizzati storicamente alla luce della sua visione - mutata nel corso del tempo - dell'imperialismo britannico, della disoccupazione degli anni Trenta, della Guerra civile spagnola e della Seconda guerra mondiale.

British counterinsurgency: from Palestine to Northern Ireland ${ }^{3}$ era già caratterizzato dall'approccio critico e revisionista che marca così fortemente anche The Blood Never Dried: A People's History of the British Empire. L'autore si concentra sulle campagne di repressione delle rivolte locali portate avanti dalla Gran Bretagna dal 1945 in poi in Palestina, Malesia, Kenya, Cipro, Yemen del Sud e Irlanda del Nord. Già in questo volume Newsinger sfidava apertamente il tono trionfalistico della letteratura più recente, sostenendo in maniera convincente come i successi della Gran Bretagna nel portare a termine campagne controinsurrezionali, fossero dovuti non tanto a particolari abilità militari, quanto alla capacità di crearsi da parte britannica un appoggio politico a livello locale. In particolare, il volume si sofferma sulla brutalità con cui gli inglesi affrontarono la rivolta Mau Mau in Kenya negli anni Cinquanta e Sessanta, contribuendo così alla ridefinizione della storiografia corrente sull'argomento.

The Blood Never Dried: A People's History of the British Empire ${ }^{4}$, edizione originale de Il libro nero dellimpero britannico, affronta anch'esso il tema della storia dell'impero. Il libro in Gran Bretagna è stato ristampato nel 2010; la seconda edizione a partire dalla quale è stata ricavata la traduzione italiana - è uscita nel 2013. La nuova edizione aggiornata, nondimeno, sposta i limiti cronologici presi in esame fino all'amministrazione Obama evidenziando il rapporto "speciale" della Gran Bretagna con gli Stati Uniti e toccando anche il tema dei riflessi della politica britannica sulle primavere arabe.

Il libro nero dellimpero britannico è, anzitutto, una replica polemica all'apologia dell'impero britannico portata avanti da alcuni tra i più famosi storici che si sono occupati dell'argomento: Niall Ferguson, Max Boot e Robert Kaplan. Newsinger adotta un approccio che si concentra sul tema della resistenza all'impero britannico, denunciando la politica di violenza perpetrata nel mondo sotto le insegne imperiali. Il

American working class in the 1930s, London, Bookmarks, 2012; ID., Jim Larkin and the great Dublin lockout of 1913, London, Bookmarks Publications, 2013.

${ }^{2}$ NEWSINGER, John, Orwell's politics, New York : St. Martin's Press, 1999.

3 ID., British counterinsurgency : from Palestine to Northern Ireland, New York, Palgrave, 2002.

4 ID., The blood never dried: a people's history of the British Empire, London, Bookmarks, 2006. 
libro può dunque essere considerato come una storia della repressione britannica $\mathrm{o}$, più precisamente, una storia della resistenza al colonialismo britannico.

Gli autori classici che si occupano di imperialismo britannico, secondo Newsinger, ne sottacciono gli aspetti più deteriori. L'autore stigmatizza in modo particolare il revisionismo di Ferguson e di chi rivendica acriticamente la grandezza dell'impero britannico:

Nel 2003 Niall Ferguson diede alle stampe il suo Impero. Come la Gran Bretagna ha fatto il mondo moderno, un volume inteso a catturare lo spirito dei tempi. In quei giorni la politica imperiale veniva celebrata quale vero e proprio dovere che si impone che s’impone alle grandi potenze nel suo rapporto con i popoli più deboli5.

Uno degli intenti del volume è quello di offrire una risposta a questa riscrittura della storia, condizionata in modo particolare - secondo l'autore - dallo spirito dei tempi, permeato dalla "Guerra al terrorismo": una delle conseguenze che ha prodotto, infatti, è stato un tentativo di reinventare la storia dell'Impero britannico. Per giustificare le azioni del governo americano e di quello britannico era importante, sottolinea Newsinger, mostrare la benevolenza passata degli imperi nei confronti delle popolazioni assoggettate. L'intento dell'autore - ben esplicitato dal sottotitolo originale dell'opera A People's History of the British Empire - era quello di scrivere una storia "dal basso", sia in termini di prospettiva di classe, sia analizzando il punto di vista dei popoli sottoposti all'Impero britannico; per fare ciò, l'autore offre una precisa disamina dei crimini britannici, non tutti universalmente noti. Lo fa prendendo in esame luoghi e momenti della vasta storia del colonialismo britannico.

Gli storici britannici - rimarca Newsinger - amano soffermarsi sull'abolizione della schiavitù nel possedimenti britannici, ma vengono citate molto meno frequentemente le rivolte di schiavi nei possedimenti caraibici inglesi, oggetto del primo capitolo ${ }^{6}$.

L'autore non offre una lettura benigna dell'amministrazione britannica neppure prendendo in esame la devastante carestia irlandese delle patate del 1840 , che uccise un milione di persone: la responsabilità, a suo giudizio, risiederebbe tutta nelle miopi e indifferenti politiche dell'amministrazione inglese ${ }^{7}$.

Newsinger per avvalorare questa teoria si serve delle parole del repubblicano John Mitchel, che aveva evidenziato come in «in ognuno di quegli anni, '46, '47 e '48,

5 NEWSINGER, John, Il libro nero dell'impero britannico, Palermo, 21 editore, 2014, p. 24.

${ }^{6}$ Ibidem, pp. 30-56.

7 Ibidem, pp. 57-75. 
l'Irlanda avesse continuato a esportare cibo in Inghilterra per un valore di 15 milioni di sterline» ${ }^{8}$.

Il terzo capitolo è dedicato alla guerra dell'oppio. Newsinger, dopo avere definito l'impero britannico come «il più grosso spacciatore di droga che il mondo abbia mai ricevuto»9, sottolinea come il contrabbando di oppio sia sottaciuto da alcune delle più importanti opere sull'Impero britannico.

Il quarto e l'ottavo capitolo trattano dell'India; il quinto e il nono si occupano invece dell'Egitto. In entrambi i casi Newsinger passa in rassegna le reticenze della storiografia sull'imperialismo britannico.

Il sesto capitolo è focalizzato sul periodo interbellico e sulla crisi dell'Impero:

Gli inglesi si ritrovarono a dover affrontare focolai di rivolta in Irlanda, Egitto, India, Mesopotamia (Iraq) e Cina, il tutto in un momento in cui il governo nutriva forti timori che nella stessa Gran Bretagna potesse scoppiare una rivolta popolare, dacché la rivoluzione bolscevica aveva profondamente mutato il contesto internazionale ${ }^{10}$.

Il capitolo settimo è incentrato sulla rivolta araba in Palestina. La lettura di Newsinger della politica britannica riguardo alle rivendicazioni dei sionisti sulla Palestina è netta: «La Gran Bretagna considerò il collegamento con i sionisti un mezzo per rafforzare le rivendicazioni sulla Palestina. Se gli inglesi avessero deciso di sponsorizzare i sionisti questa scelta avrebbe di fatto neutralizzato le rivendicazioni della Francia sul paese» ${ }^{11}$.

La rivolta Mau Mau in Kenya - oggetto del capitolo decimo - è stata per lungo tempo insabbiata e dimenticata da parte della storiografia. Newsinger ne rievoca le atrocità e individua una linea di continuità tra la sanguinosa decolonizzazione del Kenya e l'altrettanto sanguinosa conquista ${ }^{12}$.

Il capitolo undicesimo è dedicato alla presenza britannica in Oriente: alle feroci campagne anti-insurrezionali contro i malesi nel 1940 e in particolare ai metodi innovativi attuati dal generale Briggs per piegare la resistenza comunista (l'isolamento della popolazione contadina cinese in "nuovi villaggi" recintati e sorvegliati).

L'accurata critica di Newsinger si sofferma negli ultimi due capitoli sul sostegno alle politiche imperialiste offerto dal partito laburista, individuando così una linea di

\footnotetext{
8 Ibidem, p. 69.

9 Ibidem, p. 76.

${ }^{10}$ Ibidem, p. 142

${ }^{11}$ Ibidem, p. 169.

12 Ibidem, p. 246.
} 
continuità che contribuisce a spiegare il sostegno di Blair alla guerra di aggressione contro l'Iraq.

Il libro di Newsinger si conclude con una riflessione sui nuovi aspetti dell'imperialismo, proponendo una chiave di lettura per spiegare come la Gran Bretagna si sia così intimamente legata al nuovo impero americano. L'autore sostiene che non si tratti di qualcosa di nuovo: questa caratteristica della politica del governo laburista si è sviluppata durante il corso degli anni. Newsinger esprime in chiusura del volume la speranza che coloro che resistono ai tentativi di neo-colonialismo possano riuscire a portare a termine efficacemente la loro resistenza; l'evidenza storica sostiene l'autore - mostra come gli imperi, benché operino in maniera brutale, siano contraddistinti da una certa fragilità.

Il libro si distingue dunque dal resto della letteratura sul tema dell'imperialismo britannico per il suo sguardo critico sul sistema imperiale. Il volume è una riuscita galleria degli spaccati più cruenti della repressione all'interno dell'Impero britannico; si tratta di un testo fortemente militante, attitudine rivendicata a più riprese dall'autore. Uno dei meriti indiscutibili del libro di Newsinger è quello di rappresentare una voce fuori dal coro in ragione della sua prospettiva: l'autore evidenzia non tanto il fatto che l'impero sia stato realizzato attraverso le guerre, ma come sia stato mantenuto in essere attraverso una sanguinosa serie di repressioni, sino agli ultimi giorni della sua esistenza.

Il [...] volume intende mostrare come [...] l'inevitabile realtà del colonialismo e più specificamente - come uno sguardo ravvicinato sulla storia dell'Impero britannico finisca con il rivelare episodi non meno brutali di quelli che punteggiano la storia di tutti gli altri imperi'13.

Newsinger intende sottolineare la mostruosità e l'anomalia degli imperi e lo fa in modo fortemente militante lanciando un j’accuse nei confronti della politica labour, considerata anch'essa fortemente imperialista ${ }^{14}$, e criticando la subordinazione britannica all'imperialismo militare americano.

Dispiace non trovare un indice dei nomi, che sarebbe di sicura utilità - anche in considerazione della struttura del libro - per orientarsi in una ricerca puntuale di luoghi o persone all'interno del volume.

13 Ibidem, p. 24.

14 Ibidem, pp. 28-29. 


\section{* L'autore}

Jacopo Bassi ha conseguito la laurea specialistica in Storia d'Europa presso l'Università degli Studi di Bologna. Nel corso dei suoi studi si è occupato di storia della Grecia e dell'Albania in età contemporanea e di storia e istituzioni della Chiesa ortodossa. Ha lavorato per le case editrici Éditions des femmes, Il Mulino e Zanichelli.

URL: < http://www.studistorici.com/2009/02/24/jacopo_bassi/ >

\section{Per citare questo articolo:}

BASSI, Jacopo, «Recensione: John NEWSINGER, Il libro nero dell'Impero britannico, Palermo, 21 editore, 2015, 387 pp.», Diacronie. Studi di Storia Contemporanea : Le dittature militari: fisionomia ed eredità politica, 29/12/2015, URL:< http://www.studistorici.com/2015/12/29/bassi_numero_24/ >

\section{Diacronie Studi di Storia Contemporanea $\approx$ www.diacronie.it}

Risorsa digitale indipendente a carattere storiografico. Uscita trimestrale. redazione.diacronie@hotmail.it

Comitato di redazione: Jacopo Bassi - Luca Bufarale - Elisa Grandi - Antonio César Moreno Cantano - Deborah Paci - Fausto Pietrancosta - Alessandro Salvador - Matteo Tomasoni - Luca Zuccolo

Diritti: gli articoli di Diacronie. Studi di Storia Contemporanea sono pubblicati sotto licenza Creative Commons 3.0. Possono essere riprodotti e modificati a patto di indicare eventuali modifiche dei contenuti, di riconoscere la paternità dell'opera e di condividerla allo stesso modo. La citazione di estratti è comunque sempre autorizzata, nei limiti previsti dalla legge. 